\title{
Performance Analysis of Cyclic Prefix and Zero Padding Equalization Technique for MIMO-OFDM System
}

\author{
Preeti Sondhi and Vinit Grewal* \\ ECE \\ Guru Nanak Dev University \\ Jalandhar, Punjab, India \\ sondhipreeti13@gmail.com, vinitdhaliwal19@gmail.com
}

\begin{abstract}
The ever increasing development in wireless cellular mobile system for future generation is that by using MIMO architecture, it is possible to increase the system capacity, higher bit rate, smaller bit error rates and reliability. Moreover, OFDM has become one of the popular modulation techniques to mitigate the effects of multipath fading by providing orthogonality between flat narrowband channels. In a typical OFDM broadband wireless communication system, a guard interval (GI) is inserted to avoid the inter-symbol interference (ISI) and equalizer is required to mitigate the inter-carrier interference (ICI). Without GI, many time-delayed visions of the transmitted waveform would interfere with each other. Thus, MIMO-OFDM is a powerful modulation technique used for high data rate and is able to eliminate inter-symbol interference by using guard interval and inter-carrier interference by using an equalizer.

The fundamental goal of this paper is to analyze equalization technique for MIMOOFDM system for the uplink in broadband wireless communication system. An equalization technique is proposed for general MIMO OFDM systems for rayleigh fading channel in this paper. This paper is devoted to study the comparison between CP and ZP MIMO-OFDM systems with and without the use of equalization technique system in terms of BER versus $E_{b} N_{o}$ plots for Rayleigh fading channel. There are several options for GI. One choice of GI is zero padding (ZP). In this scheme, no waveform is transmitted in the GI duration. However, the zero-padded waveform would destroy the orthogonality of subcarriers and results in ICI. The cyclic prefix $(C P)$ is a good substitute of the zeropadded GI waveform. CP is a crucial feature of OFDM to combat the effect of multipath in which an end-portion of waveform is copied and inserted at the beginning of waveform.

Transmission of symbol with CP/ZP reduces the ISI and Bit Error Rate (BER). Furthermore, BER performance for cyclic prefix and zero padding for MIMO-OFDM systems with and without the use of equalization technique has also been compared in this paper. The future challenges are also highlighted in this paper.
\end{abstract}

Keywords: MIMO-OFDM, ISI, ICI, GI, CP, ZP

\section{Introduction}

The two important impairments of wireless mobile communication systems are fading and interference caused by many factors like multipath propagation and relative motion between transmitter and receiver.

OFDM system has become an attractive technique for broadband communications to combat the effects of multipath fading and inter-symbol interference (ISI) by inserting cyclic prefix (CP) and zero padding (ZP) between different symbols [1]. However, in order to cope with long delayed multipath, longer $\mathrm{CP}$ is need; Furthermore, large amount 
of pilots are also inserted into data frames for system synchronization and channel estimation.

A baseband OFDM system model with $\mathrm{N}$ subcarriers is shown in Figure 1 [1]. It consists of transmitter, channel and receiver blocks. At transmitter, input bit stream is first subjected to channel encoder to reduce the probability of error at receiver due to multipath fading effects. Then the symbol sequence is converted into parallel format and IFFT (OFDM modulation) is applied and again converted into serial format. At the receiver the signal is digitized using an $\mathrm{ADC}$ and the sequence is converted to parallel format and FFT (OFDM demodulation) is applied. An equalizer is used to suppress the ISI and the output is then serialized. Further channel decoding is performed to get the output bit stream.

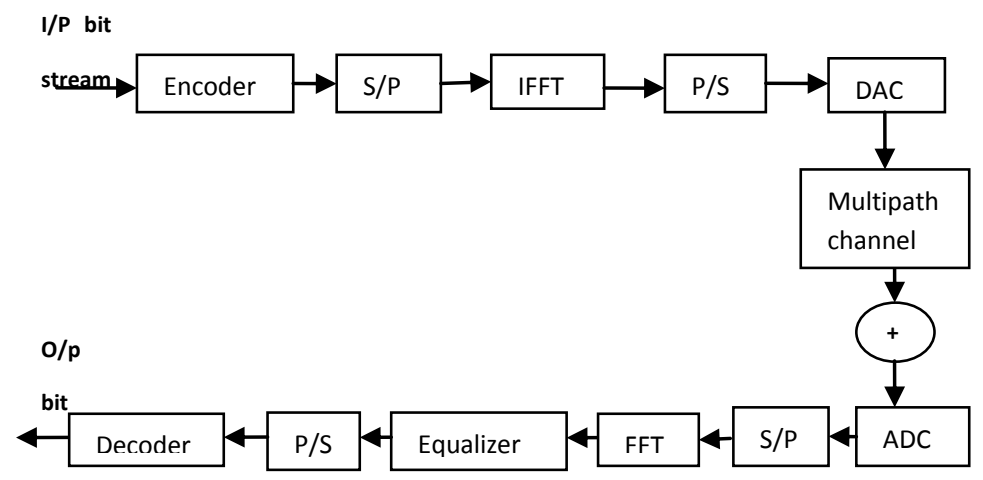

Figure 1. A Baseband Block Diagram for an OFDM System [1]

Moreover, insertion of an extra guard interval (GI) between consecutive OFDM symbols can reduce the effects of ISI but will dramatically reduce the bandwidth efficiency of the system and thus leads to low system capacity. To achieve high system capacity for multimedia applications in wireless communications, MIMO system using multiple antennas at both the transmitter and the receiver has attracted a lot of research interest due to its potential to increase the system capacity without extra bandwidth [2]. An equalizer is needed to recover a signal that suffers from ICI or ISI and helps in obtaining improved bit error rate (BER) characteristics and maintaining good signal to noise ratio (SNR).

The time variation of the channel over an OFDM block destroys the orthogonality between the subcarriers and thus introduces ICI. In addition to this, interblock interference (IBI) arises when the channel delay spread is larger than the $\mathrm{CP}$, which again results in ICI. The main purpose of using strong equalization techniques is to provide universal communication with mobility for high data rate communication. The problem of ICI can be solved by various equalization techniques proposed by various researchers which include Time-domain equalization (TEQ), Frequency-domain equalization (FEQ) and Per-tone equalization. Hence, equalization techniques are required to restore the orthogonality and so to eliminate ICI/IBI. In this paper, MIMO-OFDM system is analysed with $\mathrm{CP}$ and $\mathrm{ZP}$ as a GI in multipath environment. The motivation of this paper is to combat interference and fading effects with the help of equalization technique.

In this paper, a detailed study on MIMO-OFDM system with equalization technique for the elimination of ICI has been carried out. The rest of the paper is organised as simulation model of MIMO-OFDM transceiver model in section 2, simulation results in section 3, future scope and conclusion in section 4 and 5.

\section{Literature Survey}

In the classical parallel data systems, the total signal frequency band was divided into several subchannels to avoid overlapping of channels, but this further lead to spectrum 
inefficiency. In mid 1960s, the idea was proposed to use parallel data transmission by means of FDM with overlapping subchannels so as to fully use the available bandwidth. OFDM was introduced as an optimal version of multicarrier transmission scheme. The use of IFFT/FFT as an efficient way to realize the OFDM function and the concept of the guard interval to avoid the ISI and ICI was proposed. This proposal opened a new era for OFDM. The technique began to attract more and more attention and became very popular. Different equalization techniques had been proposed for the ICI reduction and BER performance improvement at the cost of lowering the transmission rate and reducing the bandwidth efficiency. Different approaches for reducing ICI have been proposed, including frequency-domain equalization and/or time-domain windowing. In [3] the authors propose matched-filter, least-squares (LS) and minimum mean-square error (MMSE) receivers incorporating all subcarriers. Receivers considering the dominant adjacent subcarriers have been presented in [4]. For MIMO-OFDM over doubly selective channels, a frequency-domain ICI mitigation technique is proposed in [5]. ICI selfcancellation schemes are proposed in [6] and [7]. There, redundancy is added to enable self-cancellation, which implies a substantial reduction in bandwidth efficiency. To avoid this rate loss, partial response encoding in conjunction with maximum-likelihood sequence detection to mitigate ICI in OFDM systems is studied in [8]. . The proposed work emphasized [9] that self cancellation technique was a better ICI reduction technique than the frequency offset estimation techniques for considerable computational complexity minimization. They investigated coarse frequency-offset estimation (which estimated CFO multiple of the subcarrier spacing) and fine frequency-offset estimation (which estimates $\mathrm{CFO}$ less than half the subcarrier spacing) together with self cancellation technique. The proposed self cancellation scheme was very effective in reducing the ICI when the OFDM system operated over a fast fading channel. The work presented [10] compared ZP-OFDM and CP-OFDM system in terms of nonlinearities and power backoff. The work showed that ZP-OFDM system had guaranteed symbol recovery, flexibility and improved tracking capabilities than CP-OFDM system but ZP introduced non linear distortions and need more power backoff than CP.

\section{Simulation Model of MIMO OFDM Transceiver}

A general systematic transceiver model is shown in figure 2. Initially, the incoming bit stream is mapped into data symbols using modulation technique. Then the block of data symbols is encoded into codeword matrix, which is sent through transmitting antennas in OFDM blocks. Each OFDM block consists of Nc subcarriers.

This structure corresponds to the physical layer of the IEEE 802.16 Wireless MAN OFDM air interface. The input data is generated in the form of random numbers i.e. series of ones and zeros. The length of the information bits depends upon the type of the modulation scheme used to map the bits to symbols (BPSK, QPSK, 16-QAM, 64-QAM). In MATLAB, the function randn generates the bits. The encoder used for implementation has native rate equal to $1 / 2$ and constraint length equal to 7 and the generator polynomial is given in Equations (1) and (2) to produce its two code bits [11].

$G_{1}=171_{\text {oct For } X}$

$G_{2}=133_{\text {oct For } Y}$

Puncturing operation is performed on the output of the encoder to achieve variable code rate. A punctured provides from the mother (native) code rate $1 / 2$ is performed with generator polynomials given in equations (1) and (2). Memory equal to 6 resulting in 64 states and a wide range of higher inner code rates $(2 / 3,3 / 4,5 / 6)$ can be used. In puncturing pattern, " 0 " means that the coded bit is not transmitted (i.e., punctured or masked) and "1" means that the coded bit is transmitted [12]. This code belongs to the class of so-called maximum free distance codes with constraint length seven. However, 
after puncturing, the maximum free distance, $d_{\text {free }}$, is reduced to 6 for $r=2 / 3,5$ for $r=3 / 4$, and 4 for $\mathrm{r}=5 / 6$, respectively.

A soft input maximum likelihood sequence estimator efficiently realized with the Viterbi algorithm can be employed (Proakis 1995). Deriving the soft values by taking the channel state information gives a high diversity for decoding, resulting in high performance. The number of bits that could be used for soft values is typically 3-4 bits.

The data is then fed to constellation mapper, where incoming bits are divided into groups of $i$ bits to perform modulation, which results in $2^{i}$ points. The total number of points represents a constellation. The size of $i$ for 4-PSK, 8-PSK, 16-PSK, 16-QAM and 64-QAM are 2, 3, 4, 4 and 6 respectively [13]. The mapped data symbols are then arranged in a matrix where the rows number is equal to the data subcarriers number $(N)$. In order to construct an OFDM symbol, the subcarriers are rearranged. The symbols obtained are amplitudes of certain range of sinusoids. Thus, IFFT is employed to produce a time domain signal. FFT (or IFFT) should be of length

$N_{F F T}=2$ [log2Ndata]

$N_{F F T}$ specifies the number of samples for the processing step and is always a power of 2. It is specified to be the smallest power of two, and greater than $N_{\text {used }}$ and $N$ is the number of data subcarriers.

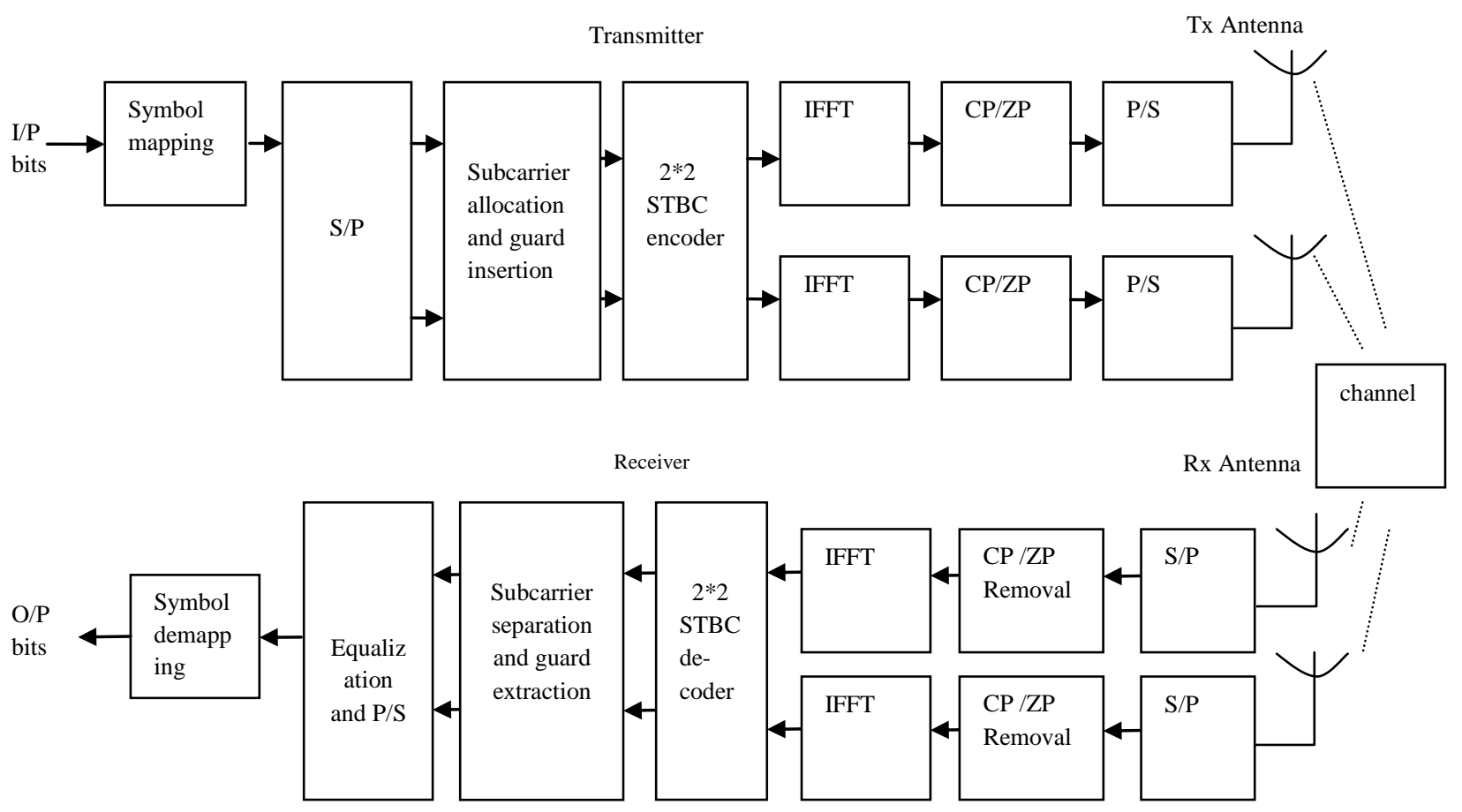

Figure 2. MIMO-OFDM Transceiver Model Adapted from [14]

The $\mathrm{t}$-th time domain sample at the $\mathrm{n}$-th subcarrier at the output of IFFT is given by $x_{t}=\sum_{0}^{N_{c}-1} X_{n} e^{\frac{j 2 \pi t n}{N}}$

Where $N_{c}$ is the number of subcarriers and $X n$ is the data symbol on the n-th subcarrier. As a result an OFDM symbol is generated.

To mitigate the effects of multipath propagation, $\mathrm{CP}$ is inserted to the time domain samples. There are four different durations of $\mathrm{CP}$ available in the standard. The ratio of $\mathrm{CP}$ time to OFDM symbol time is denoted by $\mathrm{G}$ and value for $\mathrm{G}$ can be equal to $1 / 32,1 / 6$, $1 / 8$ and $1 / 4$. CP is then added to the generated OFDM symbol to completely eliminate ISI interefernce. 
Cp_len $=$ floor $\left(0.1^{*}\right.$ block_size $)$

Where block_size is the size of each OFDM block to add CP and floor rounds the elements to the nearest integers less than or equal to the element.

Various channel models used for simulation in wireless communication system are AWGN, Rayleigh model and rician model. But for multipath, Rayleigh model is used as the most basic channel model. Only non line of sight (NLOS) components are simulated between the transmitter and receiver in this model. MATLAB provides built in function for Rayleigh channel by name 'rayleighchan'. Figure 3.15 shows OFDM signal after passing through channel.

The BER for M-ary QAM signalling For Rayleigh fading channel can be calculated is given as below

$P e=\frac{M-1}{M \log _{2} M}\left(1-\sqrt{\frac{3 \gamma \log _{2} M /\left(M^{2}-1\right)}{3 \gamma \log _{2} M /\left(M^{2}-1\right)+1}}\right)$

Where $\gamma=\left[E_{b} / N_{o}\right.$ and $\mathrm{M}$ is the modulation order.

And for QPSK theoretical BER

$P_{e=1 / 2\left[1-\frac{1}{\sqrt{1+\frac{1}{E_{b} / N_{O}}}}\right]}$

Standard Q- function defined as

$Q(x)=\frac{1}{\sqrt{2 \pi}} \int_{x}^{\infty} e^{-t^{2} / 2} d t$

If Nused subcarriers are used for carrying data out of total N (FFT size) subcarriers, the difference between time-domain SNR (SNRt) and the frequency domain SNR (SNRf) is given as follows:

SNRt $=$ SNRf $+10 \log$ Nused $/ N($ FFTsize $)[d b]$

The receiver basically performs the reverse operation as the transmitter as well as channel estimation necessary to reveal the unknown channel coefficient.

\section{Simulation Results}

By considering the table 1 simulation parameters, the results are plotted for QAM and QPSK MIMO-OFDM equalization techniques using MATLAB R2012b. The parameters values are taken from the IEEE 802.16d standards developed for OFDM.

Table 1. Simulation Parameters

\begin{tabular}{|c|l|l|}
\hline Sr. No. & Parameter & Value \\
\hline $\mathbf{1 .}$ & Number of subcarriers & 128,256 \\
\hline $\mathbf{2 .}$ & FFT size & 256 \\
\hline $\mathbf{3 .}$ & Modulation & QPSK, QAM \\
\hline $\mathbf{4 .}$ & Channel & $\begin{array}{l}\text { Rayleigh } \\
\text { fading } \\
\text { channel }\end{array}$ \\
\hline $\mathbf{5 .}$ & Number of pilot Subcarriers & 8 \\
\hline $\mathbf{6 .}$ & $\begin{array}{l}\text { Number of guard } \\
\text { Subcarriers }\end{array}$ & 256 \\
\hline $\mathbf{7 .}$ & \begin{tabular}{l} 
OFDM blocks considered \\
\hline $\mathbf{8 .}$
\end{tabular} & $\begin{array}{l}\text { Number of samples per } \\
\text { symbol }\end{array}$ \\
\hline $\mathbf{9 .}$ & Cyclic prefix length & 16 \\
\hline
\end{tabular}


The main procedure of the code contains initialization parameters and input data. The parameters that can be set at the time of initialization are the number of subcarriers, $\mathrm{CP}$ length, upsampling factor, block size, and range of the bit energy to noise rate $\left(\mathrm{E}_{b} \mathrm{~N}_{\mathrm{o}}\right)$ values (from 0 to $12 \mathrm{~dB}$ ) and channel model parameters for simulation. The input data stream is randomly generated. Output variables are available in MATLAB workspace while bit error rate (BER) values for different $\mathrm{E}_{\mathrm{b}} \mathrm{N}_{\mathrm{o}}$ are stored in text files which facilitate to draw plots. Each single block of the transmitter is tested with its counterpart of the receiver side to confirm that each block works perfectly. In this model, the simulation of the system is repeated and the number of transmitted bits and bit errors are calculated for each simulation. At the end, BER is estimated as the ratio of the total number $f$ observed errors and the total number of transmitted bits.

To combat ISI that distort transmitted signal, equalization techniques are used at the receiver. The performance evaluation can be done in terms of BER to compare the results.

Analysis of cyclic prefix- In this paper, MIMO-OFDMA system has $\mathrm{N}=128 / 256$ subcarriers, which are divided among 4 users and the CP has length $\mathrm{cp}$ len $=16$. The number of subcarriers in each OFDM symbol is $N=32$. Each user is equipped with Alamouti scheme SFC having Tx $1=2$ transmit antenna and $\mathrm{T} \times 2=2$ receive antennas. We consider a fully loaded system and therefore 32 subcarriers are assigned to each user and 16 subcarriers are assigned to each transmit antenna. The frequency selective fading channel responses are randomly generated with a Rayleigh probability distribution. The upsampling factor $(K)$ is set to 1 . The channel between transmit and receive antenna pair is assumed to be Rayleigh fading channel in this simulation model because Rayleigh fading channel has constant attenuation.

Result shows that there is an improvement in BER performance for MIMO-OFDM system with the use of equalization technique. Thus, it is possible to mitigate the effect of multipath fading and avoid ICI with the help of an equalization technique.

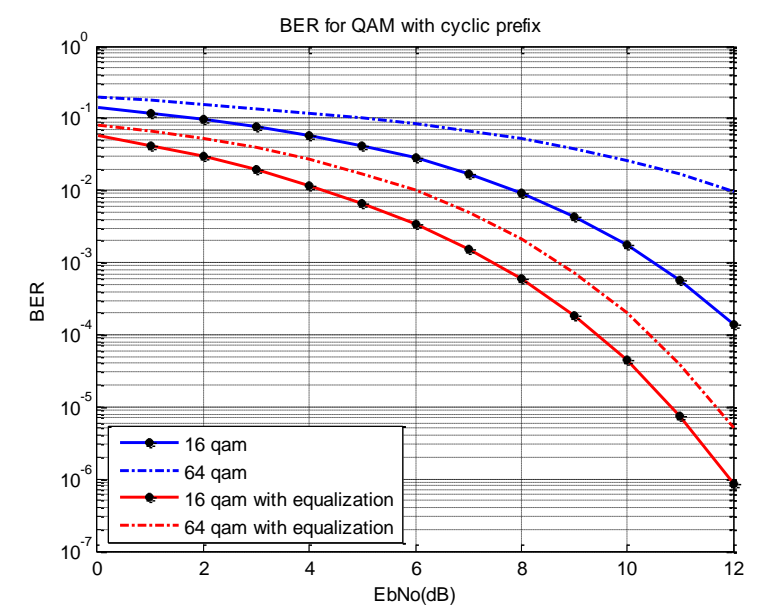

Figure 3. BER v/s SNR for QAM MIMO-OFDM with and without Equalization with Cyclic Prefix 


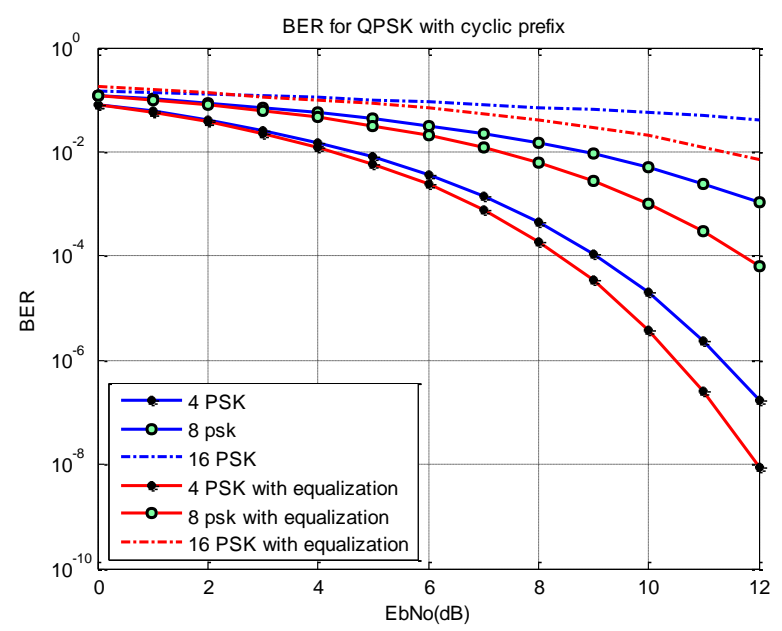

Figure 3. BER v/s SNR for QPSK MIMO-OFDM with and without Equalization in Case of Cyclic Prefix

Table 2. SNR Improvement with Equalization Technique

\begin{tabular}{|c|c|c|}
\hline Modulation Scheme & At BER & $\begin{array}{c}\text { SNR } \\
\text { Improvement }\end{array}$ \\
\hline 4-PSK & $10^{-3}$ & $1 \mathrm{db}$ \\
\hline 8-PSK & $10^{-3}$ & $2 \mathrm{db}$ \\
\hline 16-PSK & $10^{-2}$ & $4 \mathrm{db}$ \\
\hline 16-QAM & $10^{-2}$ & $4 \mathrm{db}$ \\
\hline 64-QAM & $10^{-2}$ & $6 \mathrm{db}$ \\
\hline
\end{tabular}

Analysis of zero padding- Zeroes are inserted into the guard interval instead of CP. Since the ZP is filled with zeros, the actual length of an MIMO-OFDM symbol containing $\mathrm{ZP}$ is shorter than that of an MIMO-OFDM symbol containing CP. Thus, the length of a transmission window also becomes shorter, so that the corresponding sinc-type spectrum may be wider.

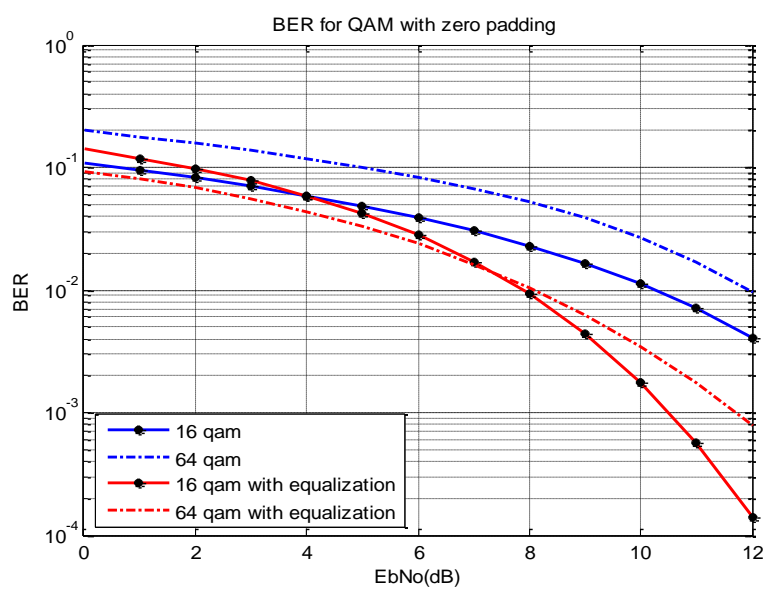

Figure 4. BER v/s SNR for QAM MIMO-OFDM with and without Equalization in Case of Zero Padding 
This implies that compared with an MIMO-OFDM symbol containing CP, an MIMOOFDM symbol containing ZP has PSD (Power Spectral Density) with the smaller in to band ripple and the larger out-of-band power. Therefore, $\mathrm{ZP}$ allows more power to be used for transmission with the peak transmission power fixed.

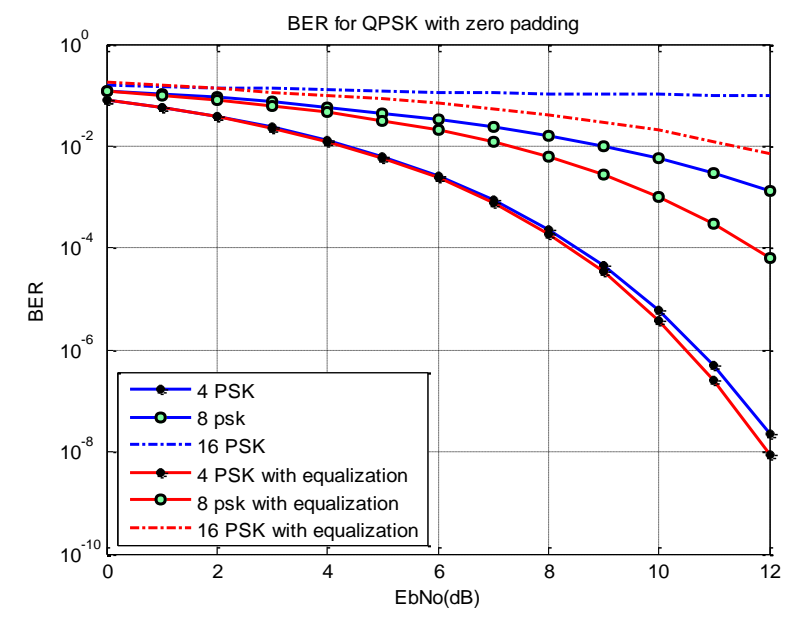

Figure 5. BER v/s SNR for QPSK MIMO-OFDM with and without Equalization in Case of Zero Padding

Result shows that MIMO-OFDM system with $\mathrm{CP}$ as a GI has superior BER performance as compared to MIMO-OFDM system with ZP as a GI. Moreover the system obtains optimum performance with the use of equalization technique.

It is clear from figure 6 that the MIMO transmission has superior performance improvement by about $4 \mathrm{~dB}$ compared with the SISO case at $10^{-4} \mathrm{db}$ in case of QAM and same improvement at $10^{-2} \mathrm{db}$ in case of QPSK. Thus, OFDM is combined with MIMO to obtain high system capacity and improved the performance of the system. The simulation results directly follows the "Shannon-Fanon's Channel Capacity Theorem", which states that as SNR or diversity increases, the channel capacity increases for a fixed bandwidth.

Table 3. SNR Improvement with Equalization Technique in Case of Zero Padding

\begin{tabular}{|c|c|c|}
\hline $\begin{array}{c}\text { Modulation } \\
\text { scheme }\end{array}$ & At BER & $\begin{array}{c}\text { SNR } \\
\text { Improvement }\end{array}$ \\
\hline 4-PSK & $10^{-7}$ & $0.2 \mathrm{db}$ \\
\hline 8-PSK & $10^{-3}$ & $2 \mathrm{db}$ \\
\hline 16-PSK & $10^{-1}$ & $4 \mathrm{db}$ \\
\hline 16-QAM & $10^{-2}$ & $2 \mathrm{db}$ \\
\hline 64-QAM & $10^{-2}$ & $4 \mathrm{db}$ \\
\hline
\end{tabular}




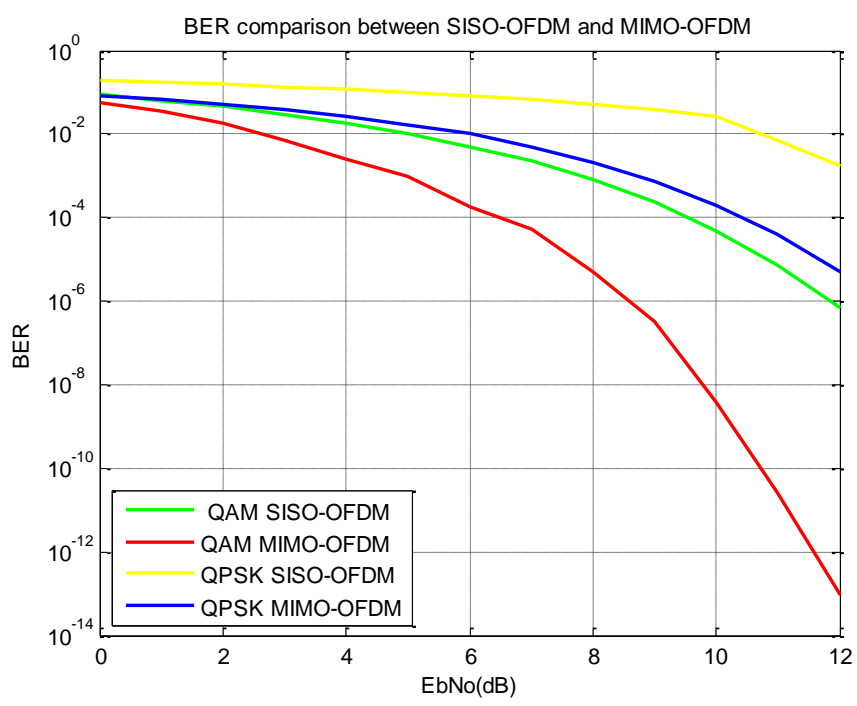

\section{Figure 6. BER Performance Comparison between SISO-OFDM and MIMO- OFDM for QAM and QPSK}

\section{Open Issues and Future Scope}

An ample of research has been done on this topic before. But there is a lot of scope for researchers to work on the issues in this direction. Based on the literature survey, the various issues and challenges have been discussed below. CP or ZP plays an important role in combating multipath effects by reducing ICI to maintain orthogonality between subcarriers and eliminating the ISI. It is also the fact that inserting CP or ZP has its own cost, a part of signal energy is lost since $\mathrm{CP}$ or $\mathrm{ZP}$ carries no information. Length of $\mathrm{CP}$ or $\mathrm{ZP}$ depends on maximum channel delay. Therefore in future, it may be possible to "measure" the channel maximum delays and insert the $\mathrm{CP}$ or $\mathrm{ZP}$ according to the measurement. Dynamic length of CP or ZP may be improve the Performance of OFDM system for that we need to develop the technique to select the length of $\mathrm{CP}$ or $\mathrm{ZP}$ as per maximum channel delay.

In future work, MIMO can be combined with orthogonal frequency and code division multiple access (OFCDMA) to utilize extraordinary capacity without any increase in the bandwidth. MIMO system also has certain issues and challenges that can be solved in future. Firstly, the cellular network base stations can support limited number of mobile terminals because of having limited processing capability. The channel state information (CSI) of each mobile station needs to be known to the base station. Channel estimation errors may results in the severe performance degradation. Thus accurate channel estimation is of prime importance in MIMO communications. Another important issue is the intercarrier interference (ICI) due to multipath fading channels and inaccurate channel estimation. Moreover, multiple transmitter and receiver chains leads to increased hardware cost and increased complexity of the systems. This makes real-time implementations of near optimum multiple antenna techniques very challenging. Various detrimental effects can arise during practical implementations such as ISI effects, carrier frequency offsets, spatial correlation effects.

\section{Conclusion}

The performance of the CP and ZP MIMO-OFDM system is analyzed and verified with simulation results. Optimum BER performance was obtained in case of $\mathrm{CP}$ as compared to ZP MIMO-OFDM system. GI is used to avoid ISI. But it leads to low system capacity. Thus, OFDM is combined with the MIMO system to increase the system 
capacity without any need of extra bandwidth. It has been observed from the plots that the BER performance for different modulation schemes with equalization technique outperforms without equalization technique. Therefore, equalization is required at the receiver to compensate for multipath fading channel effects. Multipath distortion causes ISI where subsequent symbols overlap with each other. Simulation result shows that GI (CP or ZP) is inserted between successive MIMO-OFDM symbols to mitigate ISI. Transmission of symbol with CP/ZP reduces the ISI and Bit Error Rate (BER). MIMOOFDM system with $\mathrm{CP}$ as a GI has superior BER performance as compared to ZP MIMO-OFDM system. ZP guarantees the recovery of the symbol and flexibility of the system but ZP needs more power as compared to CP because of the introduction of certain non-linearity distortion. Insertion of the GI for elimination of ISI results in low system capacity. Thus, MIMO is combined with the OFDM system to obtain high data rate and increase the capacity of the system.

The comparison of QPSK and QAM MIMO-OFDM indicates that, BER is large in QPSK as compared to QAM and shows that QAM MIMO - OFDM system achieves better BER results than QPSK MIMO-OFDM for the same bandwidth efficiency. Optimum results are obtained in case of QAM MIMO-OFDM by inserting CP as a GI with the use of an equalization technique for Rayleigh fading channel.

\section{References}

[1] Yuan Fang-Li, Lin Chin-Hsien, Lin Yi-Hsien, Wu Chih-Feng and Wang, "A MIMO-OFDM Digital Baseband Receiver Design with Adaptive Equalization Technique for IEEE 802.16 WMAN", IEEE ICASSP, (2009).

[2] Paulraj, Gore, Nabar and Bolcskei, "An overview of MIMO communications A key to Gigabit Wireless", Proc. IEEE, vol. 92, no. 2, (2004) Feb, pp. 198-218.

[3] X. Cai and G. B. Giannakis, "Low-complexity ICI Suppression for OFDM Over Time- And FrequencySelective Rayleigh Fading Channels", in Proc. 36th Asilomar Conf. Signals, Systems, Computers, Pacific Grove, CA, (2002) November, pp. 1822-1826.

[4] P. Schniter, "Low-complexity Equalization of OFDM in Doubly-Selective Channels", IEEE Trans. Signal Process., vol. 52, no. 4, (2004) April, pp. 1002-1011.

[5] A. Stamoulis, S. N. Diggavi, and N. Al-Dhahir, "Intercarrier Interference in MIMO OFDM", IEEE Trans. Signal Process., vol. 50, no. 10,(2002) October, pp. 2451-2464.

[6] Y. Zhao and S. Häggman, "Intercarrier Interference Self-Cancellation Scheme for OFDM Mobile Communication Systems", IEEE Trans. Commun., vol. 49, no. 7, (2001) July, pp. 1185-1191.

[7] J. Armstrong, "Analysis of New and Existing Methods of Reducing Intercarrier Interference Due to Carrier Frequency Offset in OFDM”, IEEE Trans. Commun., vol. 47, no. 3, (1999) March, pp. 365-369.

[8] H. Zhang and Y. Li, "Optimum Frequency-Domain Partial Response Encoding in OFDM", IEEE Trans. Commun., vol. 51, no. 7, (2003) July, pp. 1064-1068.

[9] Seyedi Alireza and Saulnier Gary J., "General ICI Self-Cancellation Scheme for OFDM Systems", IEEE Transactions on Vehicular Technology, vol. 54, no. 1, (2005) January.

[10] Muquet Bertrand, Wang Zhengdao, Giannakis Georgios B., Courville Marc de and Duhamel Pierre, "Cyclic Prefixing or Zero Padding for Wireless Multicarrier transmissions", IEEE Transactions On Commun., vol. 50, no. 12, (2002) December.

[11] Fazel, K. and Kaiser, S., Editor "Multi-Carrier and Spread Spectrum System”, New York: John Wiley and Sons Ltd (2003).

[12] Mehlfuhrer, S., Caban, S., and Rupp, "Experimental Evaluation of Adaptive Modulation and Coding in MIMO WiMAX with Limited Feedback", EURASIP Journal on Wireless Communications and Networking, (2008) pp. 1-12.

[13] Azizul H., M., "Performance Evaluation of WiMAX/IEEE 802.16 OFDM Physical Layer", M.S. Thesis, Helsinki university of Technology, Espoo, Finland, (2007).

[14] C. Padmaja and Dr. B.L. Malleswari, "Review: Performance Analysis of Space-Time-Frequency MIMOOFDM Techniques", IEEE International Conference, ICAETR, (2014) Aug 1-2. 